\title{
Being born small-for-gestational-age is associated with an unfavourable dietary intake in Danish adolescent girls: findings from the Danish National Birth Cohort
}

Bach Kampmann, Freja; Grunnet, L. G.; Halldorsson, T. I.; Bjerregaard, A. A.; Granstrøm, C.; Pires, Sara Monteiro; Strøm, M.; Vaag, A. A.; Tetens, Inge; Olsen, S. F.

Published in:

Journal of Developmental Origins of Health and Disease

Link to article, DOI:

$10.1017 / \mathrm{S} 2040174418000910$

Publication date:

2019

Document Version

Peer reviewed version

Link back to DTU Orbit

Citation (APA):

Bach Kampmann, F., Grunnet, L. G., Halldorsson, T. I., Bjerregaard, A. A., Granstrøm, C., Pires, S. M., Strøm, M., Vaag, A. A., Tetens, I., \& Olsen, S. F. (2019). Being born small-for-gestational-age is associated with an unfavourable dietary intake in Danish adolescent girls: findings from the Danish National Birth Cohort. Journal of Developmental Origins of Health and Disease, 10(4), 488-496. https://doi.org/10.1017/S2040174418000910

\section{General rights}

Copyright and moral rights for the publications made accessible in the public portal are retained by the authors and/or other copyright owners and it is a condition of accessing publications that users recognise and abide by the legal requirements associated with these rights.

- Users may download and print one copy of any publication from the public portal for the purpose of private study or research.

- You may not further distribute the material or use it for any profit-making activity or commercial gain

- You may freely distribute the URL identifying the publication in the public portal 


\section{Being born small for gestational age is associated with an unfavourable dietary intake in}

Danish adolescent girls - findings from the Danish National Birth Cohort

F. B. Kampmann ${ }^{1,2,3,4}$, L. G. Grunnet ${ }^{3,4}$, T. I. Halldorsson ${ }^{1,5}$, A. A. Bjerregaard ${ }^{1}$, C.

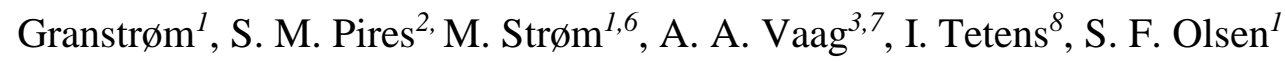

${ }^{1}$ Centre for Foetal Programming, Department of Epidemiology Research, Statens Serum Institut, Copenhagen, Denmark

${ }^{2}$ Division for Diet, Disease Prevention and Toxicology, National Food Institute, Technical University of Denmark, Kgs. Lyngby, Denmark

${ }^{3}$ Department of Endocrinology - Diabetes and Metabolism, Rigshospitalet, Copenhagen Denmark

${ }^{4}$ The Danish Diabetes Academy, Odense, Denmark

${ }^{5}$ Unit for Nutrition Research, Landspitali University Hospital and Faculty of Food Science and Nutrition, University of Iceland, Reykjavik, Iceland

${ }^{6}$ Faculty of Natural and Health Sciences, University of the Faroe Islands, Torshavn, Faroe Islands

${ }^{7}$ Cardiovascular and Metabolic Disease (CVMD) Translational Medicine Unit, Early Clinical Development, IMED Biotech Unit, AstraZeneca, Gothenburg, Sweden

${ }^{8}$ Vitality - Centre for Good Older Lives, Department of Nutrition, Sports \& Exercise, University of Copenhagen, Denmark

Corresponding authors: Freja B. Kampmann, DTU Food, Kemitorvet, building 202, Kgs. Lyngby, Denmark. Email: fredr@food.dtu.dk.

Word count: Abstract $=264$ Text $=4531$ Number of tables: 5 


\section{Abstract}

Individuals born small have an increased risk for developing type 2 diabetes. Altered food preferences in these subjects seem to play a role, however limited evidence is available on the association between being born small-for-gestational-age (SGA) at term and food intake in adolescence. Alterations in leptin, ghrelin, and dopamine levels are suggested mechanisms linking SGA with later food intake. From a large prospective Danish National Birth Cohort we compared dietary intake of adolescents born SGA with normal-for-gestational-age (NGA) adolescents. Intake of foods and nutrients was assessed by a validated food frequency questionnaire in a subsample of 15,607 14 year olds individuals born at term. SGA was defined by birth weight $(\mathrm{BW})<10^{\text {th }}$ percentile $(\mathrm{n}=1,470)$ and NGA as BW between $10-90^{\text {th }}$ percentile $(n=14,137)$ according to sex and gestational age specific BW standard curves. Girls born SGA had a 7\% (95\% CI: 3-12\%, p =0.002) higher intake of added sugar and a 2-8\% lower intake of dietary fibre, vegetables, polyunsaturated fatty acids, and total N-6, compared to NGA girls $(\mathrm{p}<0.05)$. Adjusting for parental socio-occupational status, maternal smoking and diet in pregnancy did not substantially change the differences in dietary intake, except from dietary fibre, which were no longer statistical significant. No significant differences in dietary intake between SGA and NGA boys were found. In summary, girls born SGA had an unfavourable dietary intake compared to NGA girls. These differences persisted after controlling for potential confounders, thus supporting a foetal programming effect on dietary intake in girls born SGA at term. However, residual confounding by other factors operating early in childhood cannot be excluded.

Key words: Foetal programming, small-for-gestational-age (SGA), dietary intake, adolescence 


\section{Introduction}

The developmental-origins of health and disease hypothesis propose that adverse foetal exposures may lead to permanent foetal adaptations in both anatomy and physiology. These adaptations may be beneficial for short-term foetal survival and may result in an altered longterm risk of metabolic diseases later in life ${ }^{1-3}$. Being born with a low birth weight or smallfor gestational-age (SGA) has often been used as a marker for an adverse foetal environment, and over the last two decades numerous studies have shown that individuals born small are at increased risk of developing type 2 diabetes (T2D) later in life ${ }^{4,5}$. Recently, two reviews suggested that insults in utero may impact the individual's food preferences towards energydense, less healthy foods, and further proposed that these alterations in food intake could contribute to the risk for later disease ${ }^{6,7}$. Among other things, they highlighted that several observational studies in children, young adults, and in middle-aged subjects have shown that individuals born small or exposed to famine during gestation had higher intake of palatable foods high in carbohydrate and/or fat compared to normal birth weight individuals ${ }^{8-12}$. An observational study of young Finnish adult men and women born preterm (n=307), observed that prenatal growth retardation was associated with lower intake of vegetables, fruits, and berries in young adulthood, compared to controls ${ }^{13}$. These findings are supported by animal studies of rats, showing that an adverse foetal environment can alter food preferences towards unhealthy eating habits ${ }^{14,15}$. In addition, a small human intervention study ( $\left.\mathrm{n}=16\right)$ showed that intrauterine growth restriction (IUGR) correlated with a reduced hedonic response to a sweet solution in preterm infants on their first day of life, when examining their orofacial expression, compared to controls exposed to a water solution ${ }^{16}$. This suggests that IUGR infants are less sensitive to the enjoyment of sweet taste, and therefore need an increased intake of sugar to achieve the pleasure associated with the consumption of sugar ${ }^{16}$. Thus, 
these two reviews show evidence from multiple sources for foetal programming on food preferences later in life. However, many of the studies are based upon small sample sizes and not all studies adjust for potential confounders.

The potential mechanisms by which dietary intake in childhood, youth, and adulthood are influenced by being born small are not well understood. It has been proposed that nutritional insult during foetal life may program food preferences later in life, possibly mediated by alterations in leptin, ghrelin and dopamine levels, that jointly impact the reward response to food ${ }^{6}$. These previous studies, linking an adverse intrauterine environment with an altered unfavourable food intake later in life, were of variable size ( $n=16$ to 2,036 subjects), with the largest studies focusing on food intake in adulthood ${ }^{8,10,12}$. Poor dietary habits during adolescence have been associated with an increased risk of developing obesity and T2D in adulthood ${ }^{17,18}$. This can be due to the fact that food habits in adolescence tend to be tracked into adulthood ${ }^{19}$. From a preventive point of view, insight into dietary intake recognized in adolescence is of high importance, because it enables intervention in their habitual diet before onset of disease. Since previous studies have reported sex-dependent differences in food choice and energy intake ${ }^{20,21}$ and knowing that girls and boys at adolescent age behave differently and have different attitudes towards diet ${ }^{22}$, it is relevant to take potential sexspecific differences into account when examining the association between size at birth and dietary intake later in life.

In order to fill out existing gaps in the evidence between SGA and later food intake, we took advantage of the large prospective Danish National Birth Cohort to examine this association in a general population of adolescents not exposed to extreme intrauterine conditions such as famine. More specifically, we aimed to compare food and nutrient intake in 14-year-old boys 
and girls born SGA or normal-for-gestational-age (NGA) at term, and included adjustment for potential foetal and postnatal exposures.

\section{Methods}

\section{Study population}

The study participants originate from the Danish National Birth Cohort, a large prospective cohort study of pregnant women and their offspring, which has been described in detail elsewhere ${ }^{23}$. Briefly, the Danish National Birth Cohort enrolled more than 100,000 pregnancies between 1996 and 2002. Data were collected by telephone interview in gestational weeks 12 and 30, and 6 and 18 months postpartum. Additional data from the offspring were collected at age 7, 11, and 14 using questionnaires.

When the children reached 14 years of age, approximately 70,000 children were eligible for invitation to complete a food frequency questionnaire. When the present study was performed, the 14 years follow-up study was still ongoing and had a participation rate of approximately $45 \%$, allowing the present study to include dietary data from 21,082 14-yearold offspring from the Danish National Birth Cohort. We excluded multiple births (724 twins, 8 triplets) and only included the first born child enrolled for each woman (excluding 214 younger siblings) due to a higher risk of complications and different growth pattern among twins as compared with singletons. We only included full-term births, defined as being born at gestational week 38-42 where information on birth weight (BW) was available, resulting in a study sample of 17,493 . The inclusion of full-term births only was based upon possible differential aetiology between SGA and those born small due to being born preterm. Subjects were excluded from the analysis if their reported daily dietary intake resulted in an unrealistic estimate of energy intake (set to $<2.5 \mathrm{MJ} /$ day or $>25 \mathrm{MJ} /$ day based upon the Nordic nutrition recommendation, $2012^{24}, \mathrm{n}=200$ ). Eight outliers were removed, because the self-reported 
height and bodyweight were not compliant. We further excluded subjects born large for gestational age $(n=1,678)$. Therefore, our final study sample included 15,607 subjects.

\section{Birth measures}

Date of birth and BW was extracted from the Danish Medical Birth Registry ${ }^{25}$. Information on gestational age at birth was assessed by either (I) the mothers last menstrual period, (II) based on the expected date of delivery, or (III) derived from the Danish Medical Birth Registry $^{25}$.

In order to define SGA and NGA, sex and gestational age specific BW standard curves from full-term (gestational week 38-42) singleton births offspring from the entire Danish National Birth Cohort were developed. Individuals were classified as being born SGA if their BW was below the $10^{\text {th }}$ percentile, NGA if their BW was between the $10-90^{\text {th }}$ percentile and large for gestational age if their BW were above the $90^{\text {th }}$ percentile.

\section{Assessment of dietary intake}

Information on diet was obtained from a validated semi-quantitative web-based food frequency questionnaire filled out by the 14 -year-old offspring ${ }^{26}$, and has been described in detail elsewhere ${ }^{27}$. In short, the food frequency questionnaire was designed to assess the habitual diet during the past 12 months, and consisted of 158 food items which were clustered into 8 food groups. For the nutritional calculations, we applied assumptions of standard portion sizes. Since we did not have any standard portion size specifically for adolescents available, portion sizes were derived from a Danish population of 4-75 year-old men and women, which was developed by the Danish National Food Institute ${ }^{28}$. The nutritional software program FoodCalc v.1.3 was used to convert frequencies of intake into grams per day ${ }^{29}$. Intake of nutrients was quantified based on the National Food Composition tables ${ }^{30}$. 


\section{Dietary intake}

The selection of specific dietary outcomes included in the analysis was based on a priori knowledge about foods and nutrients previously shown to be associated with the development of T2D. We included total energy intake, macronutrient intake (protein, carbohydrate (CHO) and total fat), dietary fibre ${ }^{31,32}$, added sugar ${ }^{33}$, saturated fatty acids (SFA), monounsaturated fatty acids (MUFA) and polyunsaturated fatty acids (PUFA) ${ }^{34}$. Intake of essential fatty acids, including total N-6, and N-6/N-3 ratio were also separately estimated. We further reported intake of fruits ${ }^{35}$, vegetables ${ }^{35}$, sugar sweetened beverages (SSB) ${ }^{35,36}$, and red meat ${ }^{35,37}$.

\section{Covariates}

Information on parental socio-occupational status and maternal lifestyle characteristics were derived from telephone interviews during and after pregnancy. Maternal diet during pregnancy was obtained from a food frequency questionnaire filled out by the mother at gestational week 25. From these data, a healthy eating index score of the maternal diet was calculated based upon the Danish National Food Based Dietary Guidelines. Data on waist circumference of the adolescents was obtained from the 7-year questionnaire, and current body weight $(\mathrm{kg})$ and height $(\mathrm{cm})$ of the adolescents was obtained from the food frequency questionnaire filled out at 14 years of age. All information was self-reported by the adolescent at home, except for maternal age at delivery and current age of the adolescent, which were calculated based on their date of births.

\section{Statistical analyses}

We applied general linear models to test for interaction between sex and size for gestational age on all dietary outcomes $(p<0.05$ for interaction for: MUFA, PUFA, total N-6, and 
vegetables). All analyses were conducted separately for boys and girls, to account for sexspecific associations. Since our main focus was to examine whether adolescents born SGA had higher proportional intake of the nutrients in question, dietary outcomes were expressed as continuous variables and reported in relation to total daily energy intake (e.g. fruits (g/MJ) $=$ fruits (g)/total energy intake (MJ)). Total energy intake was reported in $\mathrm{MJ} /$ day, macronutrients in energy percent ( $\mathrm{E} \%$ ), and intake of foods and nutrients as g/MJ or mg/MJ. Deviations from normality for continuous variables were assessed using histograms and boxplots. Normally distributed, continuous outcomes were expressed as mean and standard deviation (SD). All food groups, as well as total energy intake, dietary fibre, added sugar, total N-6 (for girls), and N-6/N-3 ratio (for boys) were skewed. These became normally distributed after log-transformation, and were presented as median and $10-90^{\text {th }}$ percentile, whereas categorical outcomes were presented as n (percentages).

Differences in offspring and parental characteristics between SGA and NGA were analysed using Students T-test for normally distributed continuous variables and Wilcoxon rank-sum test for skewed continuous variable. Chi-square test was used for categorical outcomes.

We applied multiple linear regression analysis to compare differences in food and nutrient intake between girls born SGA and NGA while adjusting for potentially confounding and mediating variables. Model I was adjusted for: parental socio-occupational status during pregnancy, maternal smoking during pregnancy and maternal healthy eating index score during pregnancy. Since we had data on maternal pre-pregnancy BMI, maternal weight gain during pregnancy, and duration of breastfeeding only in a sub-sample of participants, we performed a sensitivity analysis (model II) by further adding these covariates to the first model (model I). Socio-occupational status was included because it is known to influence food availability and consumption ${ }^{38}$ and is associated with SGA ${ }^{39}$. Maternal smoking during 
pregnancy is associated with reduced foetal growth, and is inversely associated with educational and economical status ${ }^{40}$. Maternal healthy eating index score was included since maternal diet during pregnancy may influence the risk of having a SGA child, and has been associated with the child's food preferences later in life ${ }^{41}$. Breastfeeding is involved in the early development of eating behaviour of the child ${ }^{41}$, and could mediate the association between size at birth and later dietary intake, why it was included in our sensitivity analysis (model II, table 5). For all regression models, assumptions of normally distributed residuals and equal variance were visualised by histogram and QQ plots. Skewed residuals became normally distributed after log-transformation, and the requirements for linear regression were met.

Statistical analyses were performed using the statistical program SAS version 9.4 (SAS Institute, 127 Cary, North Carolina, USA). All tests were 2-sided and a $p$-value $\leq 0.05$ was considered statistically significant.

\section{Results}

\section{Background characteristics}

We included 7,361 boys and 8,246 girls in our analyses, of which 659 of the boys and 811 of the girls were born SGA. Table 1 summarizes the birth and current background characteristic of these offspring. The mean BW for SGA was 2,886g for boys and 2,790g for girls and was, on average, 793g (95\% CI: -825; -761g) lower in boys and 773g (95\% CI: -800; -746g) lower in girls compared to offspring born NGA. For these term infants there were no significant differences in the mean gestational age between SGA and NGA for either boys or girls (boys: $p=0.21$, girls: $p=0.17$ ). For both boys and girls, we found that those born SGA were 
breastfed for a shorter period compared to NGA offspring, had a smaller waist circumference at 7 years of age, were shorter, weighed less, and thus had lower BMI at 14 years of age, compared to offspring born NGA (Table 1). The SGA and NGA subjects did not differ in age at the time they filled out the food frequency questionnaire.

Twice as many SGA offspring were exposed to daily maternal smoking during pregnancy compared to NGA offspring (Table 2). Additionally, SGA mothers had a lower pre-pregnancy BMI and gained less weight during pregnancy, compared to NGA mothers. These findings were similar for boys and girls. No significant differences in parental socio-occupational status at time of pregnancy between SGA and NGA subjects were observed among boys ( $p=$ 0.41), while parents of girls born SGA reported lower social-occupational status compared to parents of NGA girls $(p=0.007)$.

\section{Energy and nutrient intake}

Table 3 shows the daily energy and nutrient intake for SGA and NGA offspring, stratified by sex. For boys, no statistically significant differences between SGA and NGA in energy intake, macronutrients, or micronutrients intake were observed. We did not find any differences in energy intake, macronutrients, N-6/N-3 ratio, or SFA intake between girls born SGA and NGA. However, girls born SGA reported a 7\% (95\% CI: 3-12\%, $p=0.002)$ higher intake of added sugar. Consumption of dietary fibre, PUFA and total N-6, was 2-8\% lower in SGA girls, a finding that was statistically significant for all variables $(p<0.05)$. These differences were independent of parental socio-occupational status, maternal smoking during pregnancy, and maternal pregnancy healthy eating index score, except for differences in dietary fibre, which were attenuated after adjustment and no longer statistically significant (Table 5). Additionally in the sensitivity analysis, after adjusting for maternal pre-pregnancy BMI, 
maternal pregnancy weight gain, and duration of breastfeeding, the observed differences in SGA and NGA girls still persisted (Table 5).

\section{Food intake}

Daily food intake of selected food groups is presented in table 4. There were no significant differences in any of the food groups between boys born SGA or NGA. For girls, there were no significant differences in intake of fruit, SSB or red meat. Since, differences in intake of added sugar among the girls could not be explained by differences in SSB intake, further subanalyses of 'sweets and cakes' were performed. Independently of potential confounders, girls born SGA consumed 6\% more 'sweets and cakes' (95\% CI: 1; 11\%, $p=0.02$ ) compared to girls born NGA ((median: $5.4,10-90^{\text {th }}$ percentile: $(2.6 ; 10.0)$ g/MJ vs. (median: $5.0,10-90^{\text {th }}$ percentile: $(2.5 ; 9.4) \mathrm{g} / \mathrm{MJ})$ ) (data not shown). No statistically differences in intake were observed among boys (data not shown). Furthermore, girls born SGA reported an 8\% lower (95\% CI: $-12 ;-2 \%, p=0.004)$ intake of vegetables compared to NGA girls (Table 4). Differences in intake of vegetables remained significantly lower in SGA girls after adjustment, (Table 5). When not taking total energy intake into account, similar significant associations between SGA and food intake were obtained (data not shown).

\section{Discussion}

In this study, we observed that being born SGA is associated with an unfavourable dietary intake in adolescent girls compared to girls born NGA. These eating patterns included a relatively higher consumption of added sugar, and lower intake of dietary fibre, vegetables, and unsaturated essential fatty acids. Most of our findings persisted after adjustment for parental socio-occupational status, maternal pregnancy diet, and maternal smoking during 
pregnancy and were not related to differences in maternal pregnancy weight gain or duration of breastfeeding, indicating a "foetal programming" effect on dietary intake in girls born SGA.

\section{Sex-specific associations}

Interestingly, our findings of unfavourable dietary habits in individuals born SGA were only evident among girls. Other cohorts and animal studies have also found sex-specific differences in feeding behaviour and food intake in a BW dependent manner ${ }^{8,14,42-44}$, although no consistent pattern is apparent. Contrary to our results, some studies have shown a stronger effect of BW on food intake in boys compared to girls ${ }^{43,44}$, whereas others, like us, found that the association between IUGR and unfavourable diet and dietary behaviour was only evident among girls ${ }^{8,42}$. Speculatively, our findings could be related to differences in levels of glucocorticoids between boys and girls, since glucocorticoids stimulate intake of highly palatable food in humans ${ }^{45}$ and have been shown to be transferred to a greater extent across the placenta in females than in males rat foetuses ${ }^{46}$. From our results, it could be suggested that girls might be more sensitive to insults during foetal life in relation to programming of dietary habits in later life. Alternatively, it could also be speculated that girls are able to recall and report food intake more accurately compared to boys, since adolescent girls might pay more attention to what they eat compared to boys. This could be reflected in more accurate reporting and therefore less bias introduced by noise in the analyses among the girls.

\section{Energy and macronutrient intake}

From a foetal programming point of view, it can be hypothesized that individuals born SGA have a higher energy intake compared to NGA individuals to compensate for a foetal sparse nutritional environment. Supporting this hypothesis, previous findings have shown that 
exposure to an adverse intrauterine environment may lead to alterations in appetite regulation towards an orexigenic state, enhancing food consumption ${ }^{47}$. However, we did not find any differences in energy intake or in percentage of energy intake from fat, $\mathrm{CHO}$, or protein between adolescents born SGA and NGA. Our findings were in accordance with results from Kaseva et al., who also reported similar energy intake, percentages of energy intake from fat, CHO, and protein, assessed by a 3-day food record, in 307 young Finnish adults born preterm with a BW $<1,500$ g and NGA term-born controls ${ }^{13}$. In contrast, several other observational studies have shown higher intake from fat (E\%) in 10-year-old children ${ }^{9}$ and in middle aged adults ${ }^{10-12}$ who were born small or were exposed to famine during foetal life. Additionally to preferences for fatty-foods, severe growth restriction has also been associated with a greater CHO to protein ratio consumption in young adult women $(n=37)$ compared to non-growth restricted controls ( $\mathrm{n}=942)$ although this was not displayed in higher or lower consumption of specific foods ${ }^{8}$. In the present study, we found that higher intake of some foods and nutrients at the expense of others can result in an overall less healthy diet without affecting the total energy intake. Additionally, evidence substantiates that dietary quality of $\mathrm{CHO}$ and dietary fats, rather than quantity of these macronutrients, is more crucial in a health perspective ${ }^{34,48}$.

\section{Added sugar}

Higher intake of added sugar and lower intake of vegetables all point towards craving for more energy dense, less healthy diet in SGA girls. Limited consumption of added sugar has been recommended in the prevention of $\mathrm{T} 2 \mathrm{D}{ }^{33}$ due to its association with adverse metabolic disorders, weight gain, and obesity ${ }^{49}$. Our results showing higher added sugar consumption among SGA girls are consistent with findings from a small randomized human intervention study ( $\mathrm{n}=16)$ by Ayres et al. ${ }^{16}$. They found that the intensity of IUGR was highly and positively correlated with decreased sensitivity towards sweet taste in pre-term infants on the 
first day of life. The authors proposed that IUGR individuals need a higher consumption of sugar in order to achieve a higher degree of pleasure ${ }^{16}$. However, whether this decreased sensitivity persists beyond the first few days of life in individuals born IUGR and has long term effects on their food intake is not known. Several studies have demonstrated an increased level of fasting leptin in individuals born SGA ${ }^{50-52}$. Leptin is related to perception of sweet taste ${ }^{53}$ and subjects with elevated levels of leptin have been shown to need higher concentrations of sweeteners to detect the sweet stimuli ${ }^{54}$. Therefore, alterations in leptin levels in SGA individuals could speculatively contribute to the increased intake of added sugar observed in our study in SGA girls. Unfortunately, biological specimens were not available in the present study, and we are therefore not able to explore this further. From our sub-analyses, it seems that a higher intake of added sugar among girls born SGA, was driven by intake of 'sweets and cakes', and not by differences in intake of SSB among the two exposure groups. In contrast to these findings, an observational study has shown a positive association between BW and the intake of total sugar in middle aged adults ${ }^{12}$, and others have reported no association between IUGR and consumption of sucrose ${ }^{8}$ or calories derived from sweets ${ }^{13}$ in young adults. These studies were of variable size (n-total: 307-2,036) and diets were assessed at an older age compared to our study. Since age is known to influence food choice ${ }^{21,55}$, the diversity in results could partly reflect differences in age-range of the study populations.

\section{Fruits and vegetables}

Comparisons across studies can be challenging, however our finding of a significantly lower intake of vegetables among girls born SGA is in line with findings from the Finnish study by Kaseva et al. ${ }^{13}$. On the contrary, we did not observe an association between being born small at birth and a lower intake of fruits as has been seen in other studies ${ }^{12,13}$. Discrepancies 
between the studies examining vegetables and fruits intake could also reflect variations in how food items are clustered into food groups, and divergence in food tables and recipes used when calculating foods and nutrient intake. One example is clustering vegetables, fruits, and berries into the same food group, as was done in the study by Kaseva et al. ${ }^{13}$, compared to our study where vegetables were clustered into one food group and fruits and berries into another food group.

\section{Fatty acids}

Lower intake of essential unsaturated fatty acids, including PUFA and total N-6 further substantiate an unfavourable diet among girls born SGA. Especially, substitution of SFA for PUFA has been suggested to be protective in relation to development of T2D ${ }^{34,48}$. In contrast to our findings, higher intake of PUFA was reported in IUGR young adults compared to NGA controls in the study by Kaseva et al. ${ }^{13}$. These differences could be due to the older age group in the Finnish study, since dietary habits changes during life ${ }^{21,55}$. It could also be speculated that PUFA have been estimated more accurately among the adults using 3-day food records compared to an adolescent study population using a food frequency questionnaire and who are described as less motivated to record diet ${ }^{56}$. Other studies did not find any associations between BW and PUFA in middle aged adults ${ }^{12}$ and to our knowledge, no one has reported intake of total N-6 in these studies.

\section{Influence of age and residual confounding}

The establishment of dietary habits are complex and multidimensional, are affected pre- and postnatally by biological, social and environmental factors $6,22,38,57,58$, and change throughout the lifetime ${ }^{21,55}$. It has been suggested that parents of children born small might feed their babies differently in order to promote growth, and thereby affect food preferences in early age ${ }^{8,43}$. During adolescence, parental influence of the child's food intake seems to decline ${ }^{22}$, and 
the adolescents food habits may to a higher degree reflect a more autonomous independent food intake. The degree of influence from parents and the complex surroundings and their potential effects on the adolescent's food intake is not known in this study. Though we adjusted for available information of foetal and postnatal exposures e.g. breastfeeding and socio-occupational factors, we cannot exclude that residual confounding e.g. related to parental socio-occupational position could have influenced our findings among girls.

\section{Effect size}

The effect size in the present study may appear small, but measurements of diet may include a degree of imprecision, and the true effect size of being born SGA on dietary intake is difficult to establish. It has been suggested that even small nutritional imbalances throughout life could impact long-term health ${ }^{6,8}$. Additionally, the excess risk of T2D due to an unhealthy lifestyle in individuals born small has been found to be more pronounced in younger than older individuals ${ }^{59}$. Taken together, this further emphasizes the importance of our findings of highrisk eating behaviours among adolescent girls born SGA, in spite of an immediate modest effect size.

\section{Strengths and limitations}

The main strength of this study is the use of a large sample size including both male and female adolescents nested within a large-scale prospective cohort, representing all regions of Denmark. Furthermore, information on BW was obtained from reliable records, and gestational age and sex were taken into account when subjects were classified as being born SGA or NGA. This reduced the risk of potential misclassification within the two exposure groups, since both gender and gestational age affects BW. A limitation of this study is that we 
excluded subjects born large for gestational age. This was not to discount the increased risk of metabolic diseases in individuals born LGA, but rather because our main focus was specifically on the association between offspring born small and their dietary intake later in life. The examination of an adolescent study population can be challenging when assessing dietary intake due to their lack of motivation to report food intake ${ }^{56}$, and their unstructured eating habits ${ }^{56,57}$. However, we do believe that the use of a food frequency questionnaire to assess dietary data reduces the respondent burden, and it is an acknowledged method to rank subjects according to habitual food or nutrient intake ${ }^{60}$. The food frequency questionnaire used in our study was evaluated as valid to rank adolescents according to their food intake, with a low level of misclassification (3\%) of e.g. PUFA ${ }^{26}$. Furthermore, both energy and macronutrients intake in both exposure groups was within the expected range for this age group ${ }^{20}$, supporting the reliability of our findings.

In the present study, we used retrospective data on dietary intake, and a recall period of 1 year, which might have increased the risk of recall bias. Difficulties in recalling and estimating sweets consumed during weekends were evaluated to be challenging in the applied food frequency questionnaire ${ }^{26}$. However, the potential biases apply to the entire study population, and comparability across groups is not be compromised. Finally, we had no information on current parental socio-occupational status, and cannot exclude that the present socio-occupational position could confound the results.

In conclusion, our results suggest that being born SGA may modify food intake in adolescent girls in an unfavourable direction, which could potentially increase their risk of developing metabolic diseases later in life. However, the association we observe might be mediated through factors operating early in childhood until 14 years of age such as early feeding 
practice and there may be residual confounding from this, and other factors that warrant further investigation in future studies.

\section{Acknowledgments}

We gratefully acknowledge the adolescents and their parents for their contributions to this study.

\section{Financial Support}

The study was supported by grants from The Danish Research Council (S.F.O., grant numbers 09-067124, 09-075611); Nordea-fonden (S.F.O., grant number 02-2013-2014), the Innovation Fund Denmark (A.A.V., grant number 11-115923); the Danish Diabetes Academy supported by the Novo Nordisk Foundation; and the Technical University of Copenhagen - DTU Food.

\section{Conflict of interest}

Author AAV is employed by AstraZeneca, Mölndal, Sweden and is shareholder of Novo Nordisk A/S. No other potential conflicts of interest relevant to this article were reported.

\section{Ethical approval}

The study was performed according to the Declaration of Helsinki and the protocol was approved by the National Committee on Health Research Ethics in Denmark (H-4-2011-045). 
Written informed consent was provided by the mothers in the Danish National Birth Cohort on behalf of their children and themselves. 


\section{References}

1. Hales, C. N. \& Barker, D. J. P. The thrifty phenotype hypothesis: Type 2 diabetes. Br. Med. Bull. 60, 5-20 (2001).

2. Vaag, A. A., Grunnet, L. G., Arora, G. P. \& Brøns, C. The thrifty phenotype hypothesis revisited. Diabetologia 55, 2085-2088 (2012).

3. Gluckman, P. D., Hanson, M. a., Cooper, C. \& Thornburg, K. L. Effect of in utero and early-life conditions and adult health and disease. N. Engl. J. Med. 359, 1523-1524; author reply 1524 (2008).

4. Vaag, A. et al. Genetic, nongenetic and epigenetic risk determinants in developmental programming of type 2 diabetes. Acta Obstet. Gynecol. Scand. 93, 1099-1108 (2014).

5. Whincup, P. H. et al. Birth Weight and Risk of Type 2 Diabetes A Systematic Review. Jama 300, 2886-2897 (2008).

6. Portella, a. K. et al. Effects of in utero conditions on adult feeding preferences. J. Dev. Orig. Health Dis. 3, 140-152 (2012).

7. Molle, R. D., Bischoff, A., Portella, A. \& Silveira, P. The fetal programming of food preferences: current clinical and experimental evidence. - PubMed - NCBI. J Dev Orig Heal. Dis. 1-9 (2015).

8. Barbieri, M. A. et al. Higher Spontaneous Carbohydrate Intake in Young Women. Pediatr. Res. 65, 215-220 (2009).

9. Crume, T. L. et al. The long-term impact of intrauterine growth restriction in a diverse U.S. cohort of children: the EPOCH study. Obesity (Silver Spring). 22, 608-15 (2014). 
10. Lussana, F. et al. Prenatal exposure to the Dutch famine is associated with a preference for fatty foods and a more atherogenic lipid profile. Am. J. Clin. Nutr. 88, 1648-1652 (2008).

11. Stein, A. D., Rundle, A., Wada, N., Goldbohm, R. A. \& Lumey, L. H. Associations of gestational exposure to famine with energy balance and macronutrient density of the diet at age 58 years differ according to the reference population used. J. Nutr. 139, 1555-61 (2009).

12. Perälä, M.-M. et al. Body size at birth is associated with food and nutrient intake in adulthood. PLoS One 7, e46139 (2012).

13. Kaseva, N. et al. Diet and nutrient intake in young adults born preterm at very low birth weight. J. Pediatr. 163, 43-8 (2013).

14. Bellinger, L., Lilley, C. \& Langley-Evans, S. C. Prenatal exposure to a maternal lowprotein diet programmes a preference for high-fat foods in the young adult rat. Br. J. Nutr. 92, 513-20 (2004).

15. Krechowec, S. O., Vickers, M. H. \& Breier, B. H. Fetal programming of postnatal phenotype and obesity - experimental evidence from biomedical research. Proc. New Zeal. Soc. Anim. Prod. 64, 24-29 (2004).

16. Ayres, C. et al. Intrauterine growth restriction and the fetal programming of the hedonic response to sweet taste in newborn infants. Int. J. Pediatr. 2012, 657379 (2012).

17. Malik, V. S. et al. Dietary patterns during adolescence and risk of type 2 diabetes in middle-aged women. Diabetes Care 35, 12-18 (2012). 
18. Naja, F. et al. A Western dietary pattern is associated with overweight and obesity in a national sample of Lebanese adolescents (13-19 years): a cross-sectional study. Br. J. Nutr. 114, 1909-1919 (2015).

19. Wiium, N., Breivik, K. \& Wold, B. Growth trajectories of health behaviors from adolescence through young adulthood. Int. J. Environ. Res. Public Health 12, 1371113729 (2015).

20. Pedersen, A. N. et al. Dietary habits in Denmark 2011-2013. Main results. (National Food Institute, 2015).

21. Westenhoefer, J. Age and gender dependentprofile of food choice. Forum Nutr 57, 4451 (2005).

22. Salvy, S., Elmo, A., Nitecki, L. A., Kluczynski, M. A. \& Roemmich, J. N. Influence of parents and friends on children ' s and adolescents ' food intake and food selection 1 3. Am. Soc. Nutr. 93, 87-92 (2011).

23. Olsen, J. et al. The Danish National Birth Cohort - its background, structure and aim. Scand J Public Heal. 29, 300-307 (2001).

24. Nordic Nutrition Recommendations 2012 Integrating nutrition and physical activity. (2014).

25. Knudsen, LB; Olsen, J. The Danish Medical Birth Registry. Dan. Med. Bull. 45, 320-3 (1998).

26. Bjerregaard, A. A., Halldorsson, T. I., Kampmann, F. B., Olsen, S. F. \& Tetens, I. Relative validity of a web-based food frequency questionnaire for Danish adolescents. 
Nutr. J. 17, 9 (2018).

27. Bjerregaard, A. A., Tetens, I., Olsen, S. F. \& Halldorsson, T. I. Reproducibility of a web-based FFQ for 13- to 15-year-old Danish adolescents. J. Nutr. Sci. 5, e5 (2016).

28. Ygil, K. H. Mål, vaegt og portionsstørrelser (Dimensions, Weight and Portion sizes of Foods). (2013).

29. Lauritsen, J. Foodcalc. (2014). Available at: http://www.ibt.ku.dk/jesper/foodcalc/.

30. Data, Danish Food Composition. (2015). Available at: www.foodcomp.dk. (Accessed: 1st May 2015)

31. Hauner, H. et al. Evidence-Based Guideline of the German Nutrition Society: Carbohydrate Intake and Prevention of Nutrition-Related Diseases. 60, 1-58 (2012).

32. Ye, E. Q., Chacko, S. A., Chou, E. L., Kugizaki, M. \& Liu, S. Greater Whole-Grain Intake Is Associated with Lower Risk of Type 2 Diabetes , Cardiovascular Disease , and Weight Gain 1 - 3. (2013).

33. Mann, J. I. et al. Evidence-based nutritional approaches to the treatment and prevention of diabetes mellitus. Nutr. Metab. Cardiovasc. Dis. 14, 373-394 (2004).

34. Sylvia, H., Hamdy, O., Mohan, V. \& Hu, B. Prevention and Management of Type 2 Diabetes: Dietary Components and Nutritional Strategies. Lancet 383, 1999-2007 (2014).

35. Schwingshackl, L. et al. Food groups and risk of type 2 diabetes mellitus: a systematic review and meta-analysis of prospective studies. Eur. J. Epidemiol. (2017).

36. malik et al 2010. Sugar-Sweetened Beverages and Risk of Metabolic Syndrome and 
Type 2 Diabetes A meta-analysis. Diabetes Care 33, 2477-2483 (2010).

37. Pan, A. et al. Red meat consumption and risk of type 2 diabetes : 3 cohorts of US adults and an updated meta-analysis. Am. J. Clin. Nutr. 94, 1088-1096 (2011).

38. Riediger, N. D., Shooshtari, S. \& Moghadasian, M. H. The Influence of Sociodemographic Factors on Patterns of Fruit and Vegetable Consumption in Canadian Adolescents. J. Am. Diet. Assoc. 107, 1511-1518 (2007).

39. Räisänen, S. et al. Contribution of socioeconomic status to the risk of small for gestational age infants--a population-based study of 1,390,165 singleton live births in Finland. Int. J. Equity Health 12, 28 (2013).

40. Phung, H. et al. Risk Factors for Low Birth Weight in a Socio-Economically Disadvantaged Population: Parity, Marital Status, Ethnicity and Cigarette Smoking. Eur. J. Endocrinol. 18, 235-243 (2017).

41. Mennella, J. A., Jagnow, C. P. \& Beauchamp, G. K. Prenatal and Postnatal Flavor Learning by Human Infants. Pediatrics 107, e88-e88 (2001).

42. Silveira, P. P. et al. Preliminary evidence for an impulsivity-based thrifty eating phenotype. Pediatr. Res. 71, 293-298 (2012).

43. Shultis, W. A. et al. Does birth weight predict childhood diet in the Avon longitudinal study of parents and children? J. Epidemiol. Community Health 59, 955-60 (2005).

44. Stafford, M. \& Lucas, A. Possible association between low birth weight and later heart disease needs to be investigated further. BMJ 316, (1998).

45. Epel, E., Lapidus, R., McEwen, B. \& Brownell, K. Stress may add bite to appetite in 
women: A laboratory study of stress-induced cortisol and eating behavior. Psychoneuroendocrinology 26, 37-49 (2001).

46. Montano, M., Wang, M. \& vom Saal, F. Sex differences in plasma corticosterone in mouse fetuses are mediated by differential placental transport from the mother and eliminated by maternal adrenalectomy or stress. J. Reprod. Fertil. 99, 283-90 (1993).

47. Block, T. \& El-Osta, A. Epigenetic programming, early life nutrition and the risk of metabolic disease. Atherosclerosis 266, 31-40 (2017).

48. Salas-Salvad, J., Martinez-González, M., Bulló, M. \& Ros, E. The role of diet in the prevention of type 2 diabetes. Nutr. Metab. Cardiovasc. Dis. 21, 32-48 (2011).

49. Te Morenga, L., Mallard, S. \& Mann, J. Dietary sugars and body weight: systematic review and meta-analyses of randomised controlled trials and cohort studies. Bmj 346, e7492 (2012)

50. Hjort, L. et al. $36 \mathrm{~h}$ fasting of young men influences adipose tissue DNA methylation of LEP and ADIPOQ in a birth weight-dependent manner. Clin. Epigenetics 9, 40 (2017).

51. Phillips, D. I. W. et al. Size at birth and plasma leptin concentrations in adult life. Int. J. Obes. 23, 1025-1029 (1999).

52. Jornayvaz, F. R. et al. Low birth weight leads to obesity, diabetes and increased leptin levels in adults: the CoLaus study. Cardiovasc. Diabetol. 15, 73 (2016).

53. Shigemura, N. et al. Leptin Modulates Behavioral Responses to Sweet Substances by Influencing Peripheral Taste Structures. Endocrinology 145, 839-847 (2004). 
54. Umabiki, M. et al. The Improvement of Sweet Taste Sensitivity with Decrease in Serum Leptin Levels During Weight Loss in Obese Females. Tohoku J. Exp. Med. 220, 267-271 (2010).

55. Navarro-Allende, A., Khataan, N. \& El-Sohemy, A. Impact of Genetic and Environmental Determinants of Taste with Food Preferences in Older Adults. J. Nutr. Elder. 27, 267-276 (2008).

56. Livingstone, M. B. \& Robson, P. Measurement of dietary intake in children. Nut Proc Soc 59, 279-293 (2000).

57. Birch, L., Arbor, A., Savage, J. S. \& Ventura, A. From Infancy to Adolescence. Can J Diet Pr. Res 68, 1-11 (2009).

58. Ayres, C. et al. Exposure to maternal smoking during fetal life affects food preferences in adulthood independent of the effects of intrauterine growth restriction. J. Dev. Orig. Health Dis. 2, 162-167 (2011).

59. Li, Y. et al. Birth weight and later life adherence to unhealthy lifestyles in predicting type 2 diabetes: prospective cohort study. BMJ 351, h3672 (2015).

60. Biró, G., Hulshof, K. F. A. M., Ovesen, L. \& Amorim Cruz, J. A. Selection of methodology to assess food intake. Eur. J. Clin. Nutr. 56 Suppl 2, S25-32 (2002). 
Table 1. Characteristics of SGA and NGA boys and girls at birth and age 14

\begin{tabular}{|c|c|c|c|c|c|c|}
\hline \multirow[b]{3}{*}{ Birth data } & \multicolumn{3}{|c|}{ Boys } & \multicolumn{3}{|c|}{ Girls } \\
\hline & SGA & NGA & $P^{a}$ & SGA & NGA & $P^{a}$ \\
\hline & N (659) & $\mathrm{N}(6702)$ & & $\mathrm{N}(811)$ & N (7435) & \\
\hline Birth weight (g) & $2886(262)$ & 3680 (348) & $<.0001$ & $2790(242)$ & $3561(330)$ & $<.0001$ \\
\hline Gestational age (days) & $280.2(8.1)$ & $280.6(8.0)$ & 0.21 & $280.7(7.6)$ & $281.1(7.7)$ & 0.17 \\
\hline \multicolumn{7}{|l|}{ Breastfed (month) } \\
\hline $0-1$ & $69(13.4)$ & $422(8.7)$ & $<.0001$ & $67(11.0)$ & $475(8.4)$ & 0.01 \\
\hline $2-3$ & $60(11.6)$ & $426(8.3)$ & & 69 (11.0) & $492(7.0)$ & \\
\hline $4-6$ & $105(20.4)$ & $902(17.7)$ & & 120 (19.1) & $945(16.7)$ & \\
\hline $7-9$ & $131(25.4)$ & 1529 (29.9) & & $158(25.2)$ & $1622(28.7)$ & \\
\hline $10+$ & $151(29.3)$ & $1810(35.4)$ & & $213(34.0)$ & $2118(37.5)$ & \\
\hline \multicolumn{7}{|l|}{ Current data } \\
\hline Age (years)* & $14.1(14.0 ; 14.2)$ & $14.1(14.0 ; 14.2)$ & $0.98^{\ddagger}$ & $14.1(14.0 ; 14.2)$ & $14.1(14.0 ; 14.2)$ & $0.82^{\ddagger}$ \\
\hline Weight (kg) & 51.7 (9.7) & $55.2(10.3)$ & $<.0001$ & $49.9(8.2)$ & $53.4(8.6)$ & $<.0001$ \\
\hline Height (cm) & $166.6(8.9)$ & $170.1(8.8)$ & $<.0001$ & $162.8(6.4)$ & $165.9(6.5)$ & $<.0001$ \\
\hline BMI (kg/m²) & $18.5(2.5)$ & $19.0(2.6)$ & $<.0001$ & $18.8(2.7)$ & $19.3(2.7)$ & $<.0001$ \\
\hline Waist circumferences at 7 years $(\mathrm{cm})$ & $55.8(4.6)$ & $57.1(4.7)$ & $<.0001$ & $55.3(5.3)$ & $56.7(4.9)$ & $<.0001$ \\
\hline
\end{tabular}

SGA: small-for-gestational-age ( $<10^{\text {th }}$ percentile), NGA: normal-for-gestational-age (10-90 ${ }^{\text {th }}$ percentile), BMI: body mass index.

Data are presented as mean (SD) for normally distributed variables, median* $\left(10-90^{\text {th }}\right.$ percentile) for skewed variables and $\mathrm{n}(\%)$ for categorical variables.

$P^{a}$ : P-values are calculated using Student’s T-test, Wilcoxon rank-sum test ${ }^{\ddagger}$, or Chi-square tests. 
F. B. Kampmann et al. Born small for gestational age is associated with an unfavourable dietary intake

Table 2. Parental characteristics of the study participants stratified by sex

\begin{tabular}{|c|c|c|c|c|c|c|}
\hline \multirow[b]{2}{*}{ Parental data } & \multicolumn{3}{|c|}{ Boys } & \multicolumn{3}{|c|}{ Girls } \\
\hline & $\begin{array}{c}\text { SGA } \\
\mathrm{N}=659\end{array}$ & $\begin{array}{c}\text { NGA } \\
\mathrm{N}=6702 \\
\end{array}$ & $P^{b}$ & $\begin{array}{c}\text { SGA } \\
\mathrm{N}=811\end{array}$ & $\begin{array}{c}\text { NGA } \\
\mathrm{N}=7435 \\
\end{array}$ & $P^{b}$ \\
\hline \multicolumn{7}{|l|}{ Maternal pre-pregnancy BMI-group $\left(\mathrm{kg} / \mathrm{m}^{2}\right)$} \\
\hline$\leq 18.5$ & $56(9.0)$ & $278(4.4)$ & $<.0001$ & $58(7.5)$ & $300(4.2)$ & $<.0001$ \\
\hline 18.6-24.9 & $445(71.1)$ & 4585 (71.7) & & $569(74.0)$ & $5097(71.9)$ & \\
\hline 25.0-29.9 & $97(15.5)$ & $1156(18.1)$ & & $107(13.9)$ & $1261(17.8)$ & \\
\hline $30.0-34.9$ & $21(3.4)$ & $290(4.5)$ & & $28(3.6)$ & $322(4.5)$ & \\
\hline $35+$ & $7(1.1)$ & $83(1.3)$ & & $7(0.9)$ & $106(1.5)$ & \\
\hline Maternal weight gain during pregnancy (kg) & $13.6(5.5)$ & $15.0(5.3)$ & $<.0001$ & $13.2(5.0)$ & $14.7(5.4)$ & $<.0001$ \\
\hline Maternal age at birth (years) & $30.5(4.3)$ & $30.7(4.1)$ & 0.31 & $30.6(4.3)$ & $30.7(4.1)$ & 0.65 \\
\hline \multicolumn{7}{|l|}{ Maternal smoking during pregnancy } \\
\hline Non-smoker & $406(70.0)$ & 5325 (79.8) & $<.0001$ & $555(68.2)$ & $5878(79.4)$ & $<.0001$ \\
\hline Occasional smoker & $54(8.2)$ & $753(11.3)$ & & $87(10.8)$ & $841(11.4)$ & \\
\hline Daily smoker & $143(21.8)$ & 595 (8.9) & & $170(21.1)$ & $689(9.3)$ & \\
\hline \multicolumn{7}{|l|}{ Parental socio-occupational status in pregnancy } \\
\hline High & $158(25.0)$ & $1721(26.7)$ & 0.41 & $181(23.4)$ & $1860(25.9)$ & 0.007 \\
\hline Intermediate & $196(31.0)$ & $2161(33.5)$ & & $229(29.6)$ & $2470(34.4)$ & \\
\hline Skilled worker(s) & $173(27.3)$ & $1652(25.6)$ & & $223(28.9)$ & $1749(24.4)$ & \\
\hline Unskilled worker(s) & $39(6.2)$ & $328(5.1)$ & & $45(5.8)$ & $369(5.2)$ & \\
\hline Student(s) & $58(9.2)$ & $510(7.9)$ & & $85(11.0)$ & $645(9.0)$ & \\
\hline Not working & $9(1.4)$ & $77(1.2)$ & & $10(1.3)$ & $78(1.1)$ & \\
\hline
\end{tabular}

SGA: small-for-gestational-age $\left(<10^{\text {th }}\right.$ percentile), NGA: normal-for-gestational-age (10-90 ${ }^{\text {th }}$ percentile), BMI: body mass index.

Data are presented as mean (SD) for normally distributed variables, median* $\left(10-90^{\text {th }}\right.$ percentile) for skewed variables and $\mathrm{n}$ (\%) for categorical variables.

$P^{b}$ : P-values are calculated using Student's T-test or Chi-square tests. 
Table 3. Energy intake and daily nutrient intake pr. MJ in SGA and NGA male and female adolescents

\begin{tabular}{|c|c|c|c|c|c|c|c|c|}
\hline \multirow[b]{2}{*}{ Nutrients } & \multicolumn{4}{|c|}{ Boys } & \multicolumn{4}{|c|}{ Girls } \\
\hline & $\begin{array}{c}\text { SGA } \\
\mathrm{N}=659\end{array}$ & $\begin{array}{c}\text { NGA } \\
\mathrm{N}=6702\end{array}$ & $\begin{array}{c}\text { Mean difference } \\
\text { or } \\
\% \text { difference } \\
(95 \% \mathrm{CI}) \\
\end{array}$ & $P^{c}$ & $\begin{array}{c}\text { SGA } \\
\mathrm{N}=811\end{array}$ & $\begin{array}{c}\text { NGA } \\
\mathrm{N}=7435\end{array}$ & $\begin{array}{c}\text { Mean difference or } \\
\text { \% difference } \\
(95 \% \text { CI }) \\
\end{array}$ & $P^{c}$ \\
\hline Energy (MJ)* & $9.2(5.8 ; 14.6)$ & $9.4(5.8 ; 15.0)$ & $-2 \%(-5 ; 1 \%)$ & 0.24 & $8.4(5.2 ; 13.7)$ & $8.6(5.3 ; 13.4)$ & $0 \%(-3 ; 3 \%)$ & 0.93 \\
\hline Protein (E \%) & $15.7(2.5)$ & $15.7(2.4)$ & $-0.02(-0.24 ; 0.21)$ & 0.88 & $15.8(2.6)$ & $15.9(2.5)$ & $0.05(-0.16 ; 0.26)$ & 0.65 \\
\hline CHO (E \%) & $51.9(5.2)$ & $52.2(5.3)$ & $-0.24(-0.73 ; 0.26)$ & 0.35 & $51.6(5.0)$ & $51.5(5.3)$ & $0.15(-0.30 ; 0.60)$ & 0.51 \\
\hline Dietary fibre (g/MJ)* & $2.96(1.99 ; 4.11)$ & $2.96(2.08 ; 4.16)$ & $0 \%(-3 ; 3 \%)$ & 1.0 & $3.01(2.09 ; 4.13)$ & $3.09(2.17 ; 4.21)$ & $-2 \%(-4 ; 0 \%)$ & 0.04 \\
\hline Added sugar (g/MJ)* & $4.13(2.02 ; 8.15)$ & 4.13 (2.09; 7.59) & $1 \%(-4 ; 6 \%)$ & 0.75 & $4.01(2.06 ; 7.35)$ & $3.75(1.93 ; 6.86)$ & $7 \%(3 ; 12 \%)$ & 0.002 \\
\hline Fat (E \%) & $32.6(4.5)$ & $32.0(4.7)$ & $0.26(-0.17 ; 0.69)$ & 0.24 & $32.6(4.5)$ & $32.6(4.5)$ & $-0.19(-0.58 ; 0.19)$ & 0.32 \\
\hline SFA (g/MJ) & $3.31(0.60)$ & $3.27(0.62)$ & $0.04(-0.02 ; 0.10)$ & 0.19 & $3.33(0.60)$ & $3.27(0.59)$ & $0.03(-0.02 ; 0.08)$ & 0.24 \\
\hline MUFA (mg/MJ) & 2977 (491) & 2954 (531) & $0.03(-0.02 ; 0.08)$ & 0.23 & $3030(534)$ & 3062 (522) & $-0.04(-0.08 ; 0.01)$ & 0.09 \\
\hline PUFA (mg/MJ) & $1384(262)$ & $1383(260)$ & $0.01(-0.02 ; 0.03)$ & 0.49 & $1386(270)$ & 1439 (292) & $-0.05(-0.07 ;-0.03)$ & $<0.0001$ \\
\hline Total N-6 (mg/MJ) & 1061 (194) & 1066 (191) & $0.00(-0.02 ; 0.02)$ & 0.84 & $1039(829 ; 1307)^{*}$ & $1081(856 ; 1340)^{*}$ & $-4 \%(-5 ;-2 \%)$ & $<.0001$ \\
\hline $\mathrm{N}-6 / \mathrm{N}-3$ ratio & $4.6(3.6 ; 6.0)^{*}$ & $4.6(3.7 ; 5.9)^{*}$ & $0 \%(-2 ; 2 \%)$ & 0.73 & $4.6(0.9)$ & $4.6(0.9)$ & $-0.02(-0.10 ; 0.05)$ & 0.51 \\
\hline
\end{tabular}

SGA: small-for-gestational-age $\left(<10^{\text {th }}\right.$ percentile), NGA: normal-for-gestational-age $\left(10-90^{\text {th }}\right.$ percentile), SFA: saturated fatty acids, MUFA: mono unsaturated fatty acids, PUFA: poly unsaturated fatty acids.

Data are presented as mean (SD) for normally distributed variables, and median* $\left(10-90^{\text {th }}\right.$ Percentile) for log-transformed variables.

$P^{c}$ : Crude estimates and p-values for the difference are obtained by simple linear regression. 
Table 4. Food intake in SGA and NGA boys and girls at age 14

\begin{tabular}{|c|c|c|c|c|c|c|c|c|}
\hline \multirow[b]{2}{*}{ Food } & \multicolumn{4}{|c|}{ Boys } & \multicolumn{4}{|c|}{ Girls } \\
\hline & $\begin{array}{c}\text { SGA } \\
\mathrm{N}=659\end{array}$ & $\begin{array}{c}\text { NGA } \\
\mathrm{N}=6702\end{array}$ & $\begin{array}{c}\text { \% difference } \\
\text { (95\% CI) }\end{array}$ & $P^{d}$ & $\begin{array}{c}\text { SGA } \\
\mathrm{N}=811\end{array}$ & $\begin{array}{c}\text { NGA } \\
\mathrm{N}=7435\end{array}$ & $\begin{array}{l}\text { \% difference } \\
\text { (95\% CI) }\end{array}$ & $P^{d}$ \\
\hline Fruits (g/MJ) & $17.4(4.7 ; 43.5)$ & $17.2(5.0 ; 41.2)$ & $8 \%(-1 ; 18 \%)$ & 0.10 & $23.0(7.8 ; 53.0)$ & $23.7(8.5 ; 52.2)$ & $-2 \%(-9 ; 4 \%)$ & 0.52 \\
\hline Vegetables (g/MJ) & $18.5(7.1 ; 36.3)$ & $18.3(7.4 ; 35.4)$ & $2 \%(-4 ; 9 \%)$ & 0.56 & $23.8(9.0 ; 43.3)$ & $24.7(10.7 ; 46.1)$ & $-8 \%(-12 ;-2 \%)$ & 0.004 \\
\hline Sugar sweetened beverages (g/MJ) & $11.7(3.4 ; 45.4)$ & $12.8(3.4 ; 38.8)$ & $-2 \%(-10 ; 9 \%)$ & 0.83 & $9.6(2.5 ; 31.8)$ & $8.7(2.3 ; 29.7)$ & $7 \%(-2 ; 17 \%)$ & 0.12 \\
\hline Red meat (g/MJ) & $7.6(4.6 ; 13.6)$ & $7.7(4.3 ; 13.6)$ & $-1 \%(-6 ; 3 \%)$ & 0.56 & $8.1(4.3 ; 14.1)$ & $7.9(4.2 ; 13.8)$ & $4 \%(-1 ; 8 \%)$ & 0.10 \\
\hline
\end{tabular}

SGA: small-for-gestational-age $\left(<10^{\text {th }}\right.$ percentile), NGA: normal-for-gestational-age $\left(10-90^{\text {th }}\right.$ percentile)

Data are presented as median (10-90 ${ }^{\text {th }}$ percentile).

$P^{d}$ : Crude estimates and p-values for the difference are obtained by simple linear regression. 
Table 5. Adjusted differences in food and nutrient intake between SGA and NGA female adolescents

\begin{tabular}{|c|c|c|c|c|}
\hline \multirow[b]{3}{*}{ Food and nutrients } & \multicolumn{2}{|l|}{ Model I } & \multicolumn{2}{|l|}{ Model II } \\
\hline & Mean difference or & $P$ & Mean difference or & $P$ \\
\hline & \% difference $(95 \% \mathrm{CI})$ & & $\%$ difference $(95 \% \mathrm{CI})$ & \\
\hline Energy (MJ)* & $0 \%(-3 ; 4 \%)$ & 0.80 & $0 \%(-3 ; 3)$ & 0.76 \\
\hline Protein (E \%) & $0.06(-0.16 ; 0.27)$ & 0.61 & $0.05(-0.16 ; 0.30)$ & 0.54 \\
\hline CHO (E \%) & $0.15(-0.30 ; 0.61)$ & 0.51 & $0.02(-0.46 ; 0.50)$ & 0.92 \\
\hline Dietary fibre (g/MJ)* & $-2 \%(-4 ; 0 \%)$ & 0.07 & $-3 \%(-5 ; 0 \%)$ & 0.03 \\
\hline Added sugar (g/MJ)* & $7 \%(2 ; 12 \%)$ & 0.002 & $7 \%(3 ; 13 \%)$ & $\begin{array}{c}0.00 \\
3\end{array}$ \\
\hline Fat (E \%) & $-0.20(-0.58 ; 0.19)$ & 0.32 & $-0.08(-0.50 ; 0.33)$ & 0.69 \\
\hline SFA (g/MJ) & $0.02(-0.03 ; 0.07)$ & 0.37 & $0.04(-0.01 ; 0.01)$ & 0.11 \\
\hline MUFA (mg/MJ) & $-0.04(-0.09 ; 0.01)$ & 0.09 & $-0.03(-0.08 ; 0.02)$ & 0.27 \\
\hline PUFA (mg/MJ) & $-0.05(-0.07 ;-0.02)$ & 0.0002 & $-0.05(-0.07 ;-0.02)$ & $\begin{array}{c}0.00 \\
04\end{array}$ \\
\hline Total N-6 (mg/MJ) & $-4 \%(-5 ;-2 \%)$ & $<.0001$ & $-4 \%(-5 ;-2 \%)$ & $\begin{array}{l}<.0 \\
001\end{array}$ \\
\hline $\mathrm{N}-6 / \mathrm{N}-3$ ratio & $-0.02(-0.10 ; 0.05)$ & 0.54 & $-0.02(-0.10 ; 0.05)$ & 0.53 \\
\hline Fruits (g/MJ)* & $-1 \%(-8 ; 5 \%)$ & 0.68 & $-4 \%(-11 ; 3 \%)$ & 0.28 \\
\hline Vegetables (g/MJ)* & $-6 \%(-11 ;-1 \%)$ & 0.01 & $-6 \%(-11 ;-1 \%)$ & 0.02 \\
\hline Sugar sweetened beverages (g/MJ)* & $6 \%(-3 ; 16 \%)$ & 0.17 & $9 \%(-1 ; 19 \%)$ & 0.09 \\
\hline Red meat (g/MJ)* & $4 \%(-1 ; 8 \%)$ & 0.11 & $4 \%(-1 ; 8 \%)$ & 0.12 \\
\hline
\end{tabular}

SGA: small-for-gestational-age (<10th percentile), NGA: normal-for-gestational-age (10-90th percentile), SFA: saturated fatty acids, MUFA: mono unsaturated fatty acids, PUFA: poly unsaturated fatty acids.

Estimated differences in food intake between SGA and NGA girls are obtained by multiple linear regressions and presented as either mean differences for normally distributed residuals or as \% differences* for log-transformed data.

Model I: Adjusted for paternal socio-occupational status, maternal smoking during pregnancy, and maternal healthy eating index score during pregnancy. $\mathrm{N}=5983$.

Model II: Adjusted for paternal socio-occupational status, maternal smoking during pregnancy, maternal healthy eating index score during pregnancy, duration of breastfeeding, maternal pre-pregnancy BMI, and maternal weight gain during pregnancy. $\mathrm{N}=5417$. 
